\title{
浅析建筑电气自动化的设计要点及其注意事项
}

\author{
李大森 王常山
}

河南硕䜭建筑工程有限公司

DOI:10.18686/bd.v2i9.1694

[摘要] 建筑电气自动化是建筑工程的重要组成部分。近年来国家相继颁布了《民用建筑电气设计规范》等规章条例, 主 要是为了规范、指导建筑电气自动化施工, 保障建筑电气工程的安全使用。因此为了保障建筑电气设备的安全运行, 本文阐 述了建筑电气自动化的主要作用及其基本要求,对建筑电气自动化的设计要点及其注意事项进行了探讨分析。

[关键词] 建筑电气自动化;作用;设计;要求;设计要点;注意事项

\section{1 建筑电气自动化的主要作用}

建筑电气自动化的作用主要表现为:(1) 有效监控建筑 电气系统和设备。现代建筑的主要特点就是规模庞大、结构 复杂, 其内部电气系统组件多而复杂。传统的电气设备管理 方式, 很容易遗漏某些设备的管理工作, 带来安全隐患。而 电气自动化管理模式, 则可以对系统中的各项设备进行实 时监控, 并将控制中心下达的各项指令在短时间内传达到 各个系统中,并及时的将系统的反馈信息送到控制中心, 从 而达到高效、实时的系统管理。(2)保障建筑物的安全。建筑 物的电气系统本身就具有一定的危险性, 受到环境、人工操 作以及设备故障等因素的影响, 很容易出现系统的安全事 故。传统的电气管理模式很难对这些问题进行及时有效的 应对, 而电气自动化管理模式下, 系统中的任何异常情况都 会被实时监控到,并利用遥控模式及时处理,这样能够大大 降低维修管理工作人员在故障维修时出现的意外危险情 况。(3)提高相关系统间的联动性。利用电气自动化技术可以 将建筑物当中的各个系统结合成一个有机的整体, 并进行 统一管理。其中包括: 空调、消防、照明以及配电等系统。当 建筑物中出现管道爆裂等紧急状况时, 系统能够自动判断 出问题所在,并形成紧急的应急处理方案,适时的将灭火、 紧急照明等系统打开, 并自动控制各个子系统进行联动。

\section{2 建筑电气自动化设计的基本要求}

2.1 明确电气自动化设计目标。建筑电气自动化设计需 要对建筑电气系统的实际需求进行分析和调研, 并以此为 基础确定电气设计目标, 使其既满足用户的电气需求, 又充 分发挥了建筑功能。同时设计者还需要对建筑电气未来的 发展前景和电气系统潜在需求进行细致分析, 尽可能将建 筑电气自动化设计满足用户未来电气使用的实际需求, 减 少因建筑电气系统升级改造所造成的资源浪费, 避免对建 筑功能和结构造成的不利影响。因此, 设计者在进行建筑电 气系统设计时, 需要围绕着建筑电气自动化设计目标开展 工作, 提高建筑电气自动化设计的前瞻性。

2.2 加强电气自动化设计可行性的分析。建筑电气自动 化设计前需要科学分析和了解建筑的电气需求, 这是保证 建筑电气自动化设计科学性、有效性和适用性的基础。因此
在建筑电气自动化设计过程中, 设计者需要从建筑功能需 求和区域的实际用电需求出发, 依据建筑的主要功能和不 同的电气需求确定供配电系统的设计参数, 使建筑电气自 动化设计既满足了用户的实际电气需求, 又避免了电气资 源的浪费, 使建筑电气自动化设计更为合理和有效。例如建 筑和商用建筑在用电量和用电负荷方面存在着明显的差 异, 设计者在进行建筑电气自动化设计的过程中, 需要充分 认识到这些差异, 编制针对性的电气设计方案, 从而提高建 筑电气自动化设计方案的有效性和适用性, 在满足不同建 筑电气需求的基础上,促进我国向节约型社会方向发展。

2.3 控制电气自动化设计质量。建筑电气自动化设计质 量控制必须依据电气自动化设计的实际需求, 科学合理的 建筑电气自动化设计, 可以很好地满足建筑电气的使用要 求和安全要求。设计者在电气设计的过程中,需要遵循的基 本要求与规范要求主要为《建筑照明设计标准》、《供配电系 统设计规范》和《民用建筑电气工程设计规范》等。设计者以 这些相关规定作为电气设计的基本要求, 既可以保障建筑 电气的实用性和安全性, 又可以提高建筑电气自动化设计 的节能效果,促进我国向节约型社会方向发展。

2.4 保证电气自动化设计的经济性。建筑电气自动化设 计时不仅要保证其有效性和适用性, 而且需要注重其经济 性, 减少不必要的各种资源浪费。设计者可以在保障建筑电 气系统质量的基础上, 平衡好经济效益和投入成本之间的 比例, 以实现经济利益的最大化。

\section{3 建筑电气自动化的设计要点及其注意事项}

3.1 建筑电气自动化的设计要点分析。具体表现为:(1) 布线设计要点。建筑电气自动化系统布线时, 要注意某些线 路需要专门的导线, 如通信线路、温度湿度传感器线路、水 位浮子开关线路、流量计线路等, 它们一般需要屏蔽线, 或 者由制造商提供专门的导线。电源线与信号、控制电缆应分 槽、分管敷设; 数据显示通道(DDC)、计算机、网络控制器、网 关等电子设备的工作接地应连在其他弱电工程共用的单独 的接地干线上。智能建筑中安装有大量的电子设备, 这些设 备分属于不同的系统, 由于这些设备工作频率、抗干扰能力 和功能等都不相同,对接地的要求也不同。(2)自动化系统输 
人设备设计要点分析。输人设备应安装在能正确反映其性 能的位置, 便于调试和维护的地方。不同类型的传感器应按 设计、产品的要求和现场实际情况确定其位置: 水管型温度 传感器、蒸汽压力传感器、水流开关、水管流量计不宜安装 在管道焊缝及其边缘上开孔焊接; 风管型湿度传感器、室内 温度传感器、风汽压力传感器、空气质量传感器应避开蒸汽 放空口及出风口处; 管型温度传感器、水管型压力传感器、 蒸汽压力传感器、水流开关的安装应与工艺管道安装同时 进行; 风管压力、温度、湿度、空气质量、空气速度、压差开关 的安装应在风管保温完成后进行。(3)自动化系统输出设备 设计要点。风阀箭头、电动阀门的箭头应与风门、电动荿门 的开闭和水流方向一致; 安装前宜进行模拟动作; 电动阀门 的口径与管道口径不一致时, 应采取渐缩管件, 但阀门口径 一般不应低于管道口径二个档次, 并应经计算确定满足设 计要求; 电动与电磁调节阀一般安装在回水管上。(4)其它电 气设备设计要点。自动化系统建筑电气设备系统的监控, 使 安全使用的重要指挥中心。它是由电脑按照建筑电气自动 化系统编制好的程序进行的, 这样就可以使设计工程大大 简化, 不需要各种设备的电气联锁控制调节原理图等, 只需 要简单的监控原理图就可以满足要求。但设计人员必须编 制较为详细的监控说明软件, 还要向制造商提供各测量元 件、控制器使用的条件清单, 以便制造商选用各种元件规 格。(5)远程处理机设计要点。楼宇自动控制系统与各可重构 处理单元 RPU 之间的通信是透明的,可利用同一线路不同 的 RPU 完成同一个控制系统。一般而言, 建筑电气自动化 系统大量监控的是空调机组, 所以将 RPU 布置在机房之中 或附近, 把空调机组控制系统使用后剩余的输人输出接口 用于连接附近的水流量计、水位信号、照明控制等。

3.2 建筑电气设备自动化设计的注意事项。主要表现 为:(1)构建自动化系统架构的注意事项。在建筑电气设备自 动化设计的过程中, 应该建立自动化的系统架构,包括电气 工程及其自动化技术需要具备的功能、需要处理的问题等 等, 在自动化的系统架构中应该设置一些管理模块, 根据实
际需要, 将相应的功能设置在管理模块中; 根据这个自动化 的系统架构还可以作为后续制度建设的依据, 并且在设置 相关人员的时候, 也可以充分的发挥指导作用; 常见的模块 有数据管理、运行监控、人员管理、电力设施养护以及电气 工程管理等等。(2)选用电气自动化设备的注意事项。电气设 备自动化技术的基础就是自动化设备, 因此自动化设备选 用的合理与否将会直接影响到整个自动化技术的科学性和 效率等等。一般情况下, 可以电气工程及其自动化技术设备 分为三个类别, 第一类别是电磁开关、自动化和变压器设备 等,属于经营设备;第二类设备主要是一些传输设备、监测 设备和网络传输设备等, 具体指的是电子信号转换系统和 信息收集传递装置等等，通过第二类设备收集的信息可以 将系统的实时动态有效的反映出来; 第三类设备主要是对 系统的加工设备进行控制。(3)环境监控的注意事项。环境监 控主要是对电气工程设备的运行环境进行检测, 还应该实 时监控温度、湿度、电压和功率等等, 并且建立相应的警戒 线值, 从而对外部环境做出正确的判断, 如果外部链接发生 比较大变化的时候, 控制中心就会及时的接收到这些变化 的数据, 从而及时发出相关的指令。

\section{4 结束语}

综上所述, 随着科技的进步发展, 促进了建筑电气自动 化进步, 改善了人类生存环境, 提高了人们的生活水平。因 此为了充分发挥建筑电气设备的作用, 必须加强对其设计 的基本要求与设计要点进行分析。

\section{[参考文献]}

[1]李洪宝,刘啸洋。建筑电气设计中若千问题的思考 [J].环球市场,2017,(17):358.

[2] 石东辰. 关于住宅建筑电气设计问题的若千思考 [J].智能城市,2017,3(11):86.

[3]伍海明.高层建筑电气自动化设计要点分析 [ J]. 电 子技术与软件工程,2016,(15):167-168.

[4]吴贤安.浅谈建筑电气工程自动化设计及应用 [J]. 建材与装饰,2016,(32):83-84. 